\title{
A trigonometric double-inequality
}

\author{
Horst Alzer
}

Horst Alzer received his doctoral degree in mathematics from the University of Bonn, and his habilitation in mathematics from the University of Würzburg.

Trigonometric functions, in particular the sine and cosine functions, play a relevant rôle in physics and various branches of mathematics. In view of their importance the properties of these functions have been studied intensively. Of special interest are inequalities for trigonometric functions. In his classical book Analytic Inequalities D.S. Mitrinović dedicated a whole section to this subject. In the recent past, several authors discovered remarkable new inequalities for sine, cosine, and their relatives. Many of them appeared in the Journal of Inequalities in Pure and Applied Mathematics, in Mathematical Inequalities and Applications, and in the Journal of Inequalities and Applications.

In this note we present a double-inequality involving sine and cosine. Our work has been inspired by a short paper published by R.J. Webster [4] in 1984. He proved the elegant inequalities

$$
|\sin (\cos x)| \leq|\cos x| \leq \cos (\sin x) \quad(x \in \mathbb{R}) .
$$

See also [1] and [2]. We offer the following counterpart of (1):

Seit Jahrzehnten spielen Ungleichungen für trigonometrische Funktionen eine wichtige Rolle in der Mathematik. D.S. Mitrinović hat dem Thema in seinem Buch „Analytic Inequalities“ ein ganzes Kapitel gewidmet. In diesen Zusammenhang gehört auch die elegante Ungleichung

$$
|\sin (\cos x)| \leq|\cos x| \leq \cos (\sin x) \quad(x \in \mathbb{R}),
$$

die im Jahr 1984 von R.J. Webster veröffentlicht wurde. In der vorliegenden Arbeit stellt der Autor für beliebige reelle Werte von $x$ eine Variation der vorhergehenden Ungleichungen in der Form

$$
\alpha|\sin (\cos x)+\sin (\sin x)| \leq|\cos x+\sin x| \leq \beta(\cos (\cos x)+\cos (\sin x))
$$

vor mit den bestmöglichen Konstanten $\alpha, \beta$, welche durch $\alpha=(\sqrt{2} \sin (1 / \sqrt{2}))^{-1}$ bzw. $\beta=(\sqrt{2} \cos (1 / \sqrt{2}))^{-1}$ gegeben sind. 
Theorem. For all real numbers $x$ we have

$$
\alpha|\sin (\cos x)+\sin (\sin x)| \leq|\cos x+\sin x| \leq \beta(\cos (\cos x)+\cos (\sin x))
$$

with the best possible constant factors

$$
\alpha=\frac{1}{\sqrt{2} \sin (1 / \sqrt{2})}=1.0884 \ldots \quad \text { and } \quad \beta=\frac{1}{\sqrt{2} \cos (1 / \sqrt{2})}=0.9301 \ldots .
$$

Proof. First, we consider the left-hand side of (2) with $\alpha$ as given in (3). We establish

$$
f(x)=|\cos x+\sin x|-\alpha|\sin (\cos x)+\sin (\sin x)| \geq 0 \quad \text { for all } x \in \mathbb{R} .
$$

Since $f(x+\pi)=f(x)$, it suffices to show that $f$ is nonnegative on $[0, \pi]$. Using the formulas

$$
\cos a+\sin a=\sqrt{2} \sin \left(\frac{\pi}{4}+a\right), \quad \cos a-\sin a=\sqrt{2} \cos \left(\frac{\pi}{4}+a\right),
$$

and

$$
\sin a+\sin b=2 \sin \frac{a+b}{2} \cos \frac{a-b}{2}
$$

we obtain the representation

$$
\frac{1}{2 \alpha} f(x)=|\delta| \sin \frac{1}{\sqrt{2}}-\left|\sin \frac{\delta}{\sqrt{2}} \cos \sqrt{\frac{1-\delta^{2}}{2}}\right|
$$

with

$$
\delta=\delta(x)=\sin \left(\frac{\pi}{4}+x\right) .
$$

If $0 \leq x \leq 3 \pi / 4$, then $0 \leq \delta \leq 1$. This leads to

$$
\frac{1}{2 \alpha} f(x)=\delta \sin \frac{1}{\sqrt{2}}-\sin \frac{\delta}{\sqrt{2}} \cos \sqrt{\frac{1-\delta^{2}}{2}} .
$$

Furthermore, if $3 \pi / 4 \leq x \leq \pi$, then $0 \leq-\delta \leq 1 / \sqrt{2}$. Hence,

$$
\frac{1}{2 \alpha} f(x)=(-\delta) \sin \frac{1}{\sqrt{2}}-\sin \frac{-\delta}{\sqrt{2}} \cos \sqrt{\frac{1-(-\delta)^{2}}{2}} .
$$

This implies that we have to prove that

$$
0 \leq t \sin \frac{1}{\sqrt{2}}-\sin \frac{t}{\sqrt{2}} \cos \sqrt{\frac{1-t^{2}}{2}} \text { for } t \in[0,1]
$$

We denote the expression on the right-hand side by $g(t)$. Differentiation gives

$$
\sqrt{2}\left(1-t^{2}\right)^{3 / 2} g^{\prime \prime}(t)=\sqrt{2} t\left(t^{2}-1\right) \cos \frac{t}{\sqrt{2}} \sin \sqrt{\frac{1-t^{2}}{2}}+h\left(\sqrt{\frac{1-t^{2}}{2}}\right) \sin \frac{t}{\sqrt{2}},
$$


where

$$
h(s)=s \cos s-\sin s .
$$

Since $h(s) \leq 0$ for $s \in[0, \pi]$, we conclude from (6) that $g^{\prime \prime}(t) \leq 0$ for $t \in[0,1]$. Thus, $g$ is concave. We have $g(0)=g(1)=0$. Hence, $g(t) \geq 0$ for $t \in[0,1]$.

Now, we prove the second inequality in (2) with $\beta$ as given in (3). We show that

$$
u(x)=\beta(\cos (\sin x)+\cos (\cos x))-|\cos x+\sin x| \geq 0 \text { for all } x \in \mathbb{R} .
$$

Since $u$ is periodic with period $\pi$, we may assume that $x \in[0, \pi]$. Applying (4) and

$$
\cos a+\cos b=2 \cos \frac{a+b}{2} \cos \frac{a-b}{2}
$$

yields

$$
\frac{1}{2 \beta} u(x)=\cos \frac{\delta}{\sqrt{2}} \cos \sqrt{\frac{1-\delta^{2}}{2}}-|\delta| \cos \frac{1}{\sqrt{2}},
$$

where $\delta$ is defined in (5). If $0 \leq x \leq 3 \pi / 4$, then $0 \leq \delta \leq 1$ and

$$
\frac{1}{2 \beta} u(x)=\cos \frac{\delta}{\sqrt{2}} \cos \sqrt{\frac{1-\delta^{2}}{2}}-\delta \cos \frac{1}{\sqrt{2}} .
$$

Moreover, if $3 \pi / 4 \leq x \leq \pi$, then $0 \leq-\delta \leq 1 / \sqrt{2}$ and

$$
\frac{1}{2 \beta} u(x)=\cos \frac{-\delta}{\sqrt{2}} \cos \sqrt{\frac{1-(-\delta)^{2}}{2}}-(-\delta) \cos \frac{1}{\sqrt{2}} .
$$

Therefore, we have to show that

$$
0 \leq \cos \frac{t}{\sqrt{2}} \cos \sqrt{\frac{1-t^{2}}{2}}-t \cos \frac{1}{\sqrt{2}} \quad \text { for } t \in[0,1] .
$$

We define for $t \in(0,1]$ :

$$
v(t)=\cos \frac{t}{\sqrt{2}} \quad \text { and } \quad w(t)=\frac{1}{t} \cos \sqrt{\frac{1-t^{2}}{2}} .
$$

The function $v$ is decreasing. To prove that $w$ is also decreasing, we apply the inequality

$$
\tan y \leq y+\frac{\tan \lambda-\lambda}{\lambda^{3}} y^{3},
$$

which holds for all $\lambda$ and $y$ with $0<\lambda<\pi / 2$ and $0 \leq y \leq \lambda$. A proof is given in [3, pp. 245, 246]. We set $\lambda=1 / \sqrt{2}$. Since

$$
\frac{\tan \lambda-\lambda}{\lambda^{3}}=0.41 \ldots
$$


we get

$$
\tan y \leq y+\frac{1}{2} y^{3} \quad \text { for } y \in[0,1 / \sqrt{2}]
$$

Let

$$
y(t)=\sqrt{\frac{1-t^{2}}{2}} .
$$

Using (8) gives

$$
\begin{aligned}
\frac{2 y(t)}{\cos y(t)} w^{\prime}(t) & =\tan y(t)-\frac{2 y(t)}{t^{2}} \leq y(t)+\frac{1}{2} y(t)^{3}-\frac{2 y(t)}{t^{2}} \\
& =-\frac{2 y(t)^{5}+3 y(t)^{3}+2 y(t)}{2 t^{2}}<0
\end{aligned}
$$

Thus, $v$ and $w$ are positive and decreasing on $(0,1]$, so that we obtain

$$
v(t) w(t) \geq v(1) w(1)=\cos \frac{1}{\sqrt{2}} \quad \text { for } t \in(0,1]
$$

This settles (7). The proof of double-inequality (2) is complete.

We define

$$
P(x)=\left|\frac{\cos x+\sin x}{\sin (\cos x)+\sin (\sin x)}\right| \quad \text { and } \quad Q(x)=\frac{|\cos x+\sin x|}{\cos (\cos x)+\cos (\sin x)} .
$$

Then we have

$$
P(\pi / 4)=\frac{1}{\sqrt{2} \sin (1 / \sqrt{2})} \quad \text { and } \quad Q(\pi / 4)=\frac{1}{\sqrt{2} \cos (1 / \sqrt{2})} .
$$

This implies that the constant factors given in (3) are the best possible.

\section{References}

[1] Bensom, H.: On sin o cos and cos o sin. Math. Gaz. 67 (1983), 43-44.

[2] Haigh, G.: Another proof that $\cos (\sin x)>\sin (\cos x)$. Math. Gaz. 68 (1984), 36.

[3] Mitrinović, D.S.: Analytic Inequalities. Springer, New York 1970.

[4] Webster, R.J.: $\cos (\sin x) \geq|\cos x| \geq|\sin (\cos x)|$. Math. Gaz. 68 (1984), 37.

\section{Horst Alzer}

Morsbacher Str. 10

D-51545 Waldbröl, Germany

e-mail: H.Alzer@gmx.de 